\title{
The effects of Sevoflurane on the cardiac action potential in pigs
}

Annemie Bauters ${ }^{1}$, Stefaan Bouchez ${ }^{1}$, Patrick Wouters ${ }^{1}$, Stefan De Hert ${ }^{1}$. ${ }^{1}$ Department of Anaesthesiology, Ghent University Hospital, Belgium.

\section{Introduction}

Some reports have associated sevoflurane anaesthesia with the development of torsades de pointes (TdP).

The traditionally used surrogate marker for drug induced TdP is a prolonged QT interval on the electrocardiogram.

Actually, QT prolongation is antiarrhythmic, provided it is not contaminated by particular abnormalities in the characteristics of

ventricular repolarization.

Triangulation is one of these proarrhythmic abnormalities.

\begin{tabular}{|l} 
Triangulation \\
$\begin{array}{l}\text { slows phase } 3 \text { repolarization of the monophasic } \\
\text { action potential (MAP) }\end{array}$ \\
$\square \quad$ renders action potential (AP) more triangular \\
increases the vulnerable period of the heart
\end{tabular}

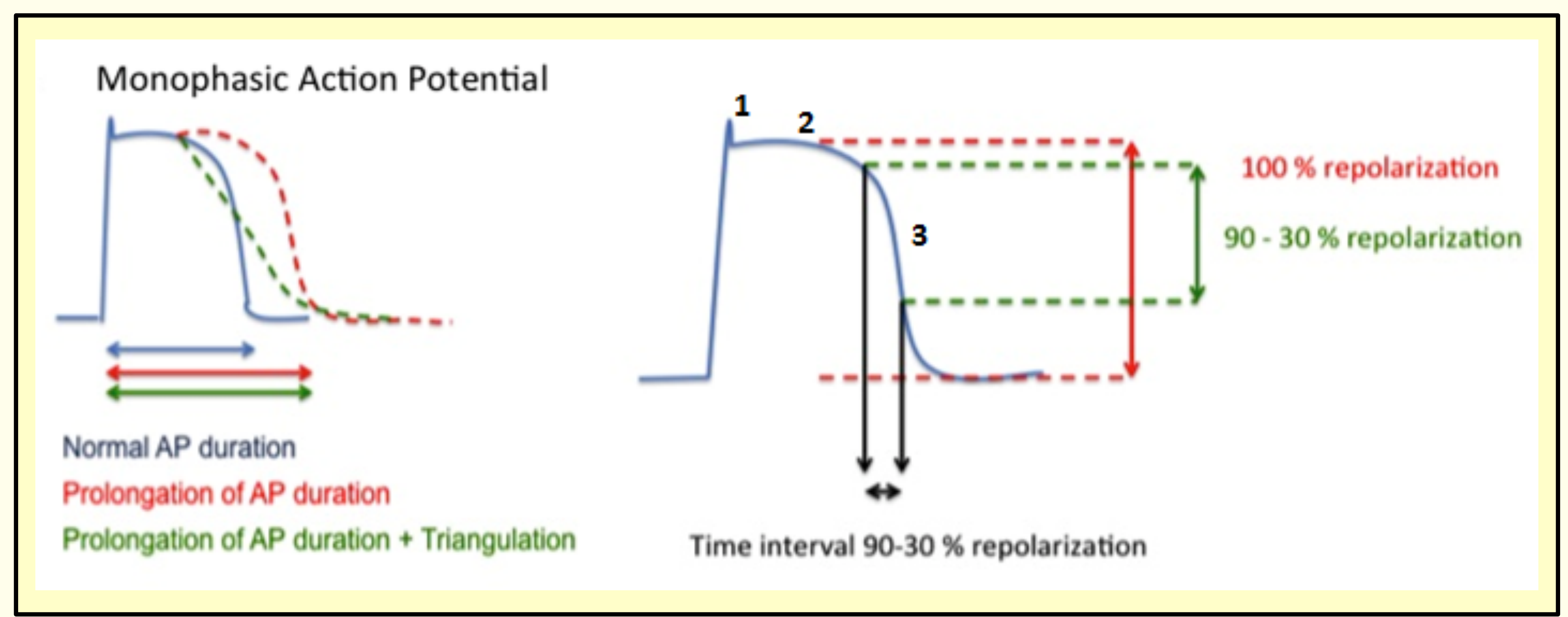

The present study investigated the effect of sevoflurane on the action potential duration (APD) and its effect on triangulation.

\section{Methods}

Research animal experiment, pilot study:

Effects of sevoflurane 4\% on APD in 5 pigs

$\square$ Pacing at a fixed heart rate

$\square$ Percutaneously introduced MAP catheter - MAP analysis with EP tracer

a MAP analysis in baseline + during administration of sevoflurane $4 \%$
Measurements:

a $\mathrm{APD}=$ time interval from the start until the end of the AP

$\square$ APD $30 / 90=$ time interval between $30 \%$ and $90 \%$ of repolarization

$=$ determines triangulation

Statistical differences were analysed using a student's t-test

\section{Results}
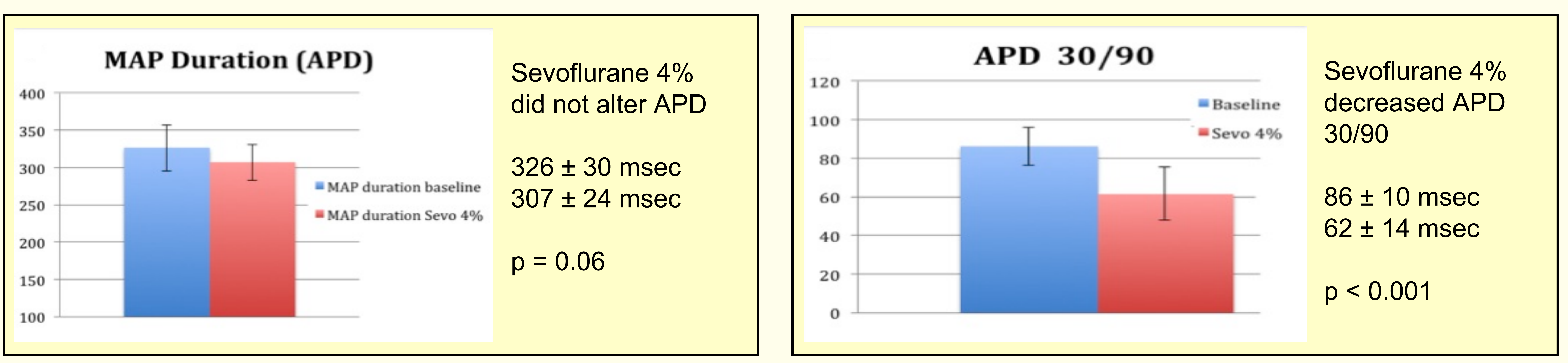

\section{Conclusion}

Triangulation is reduced when using $4 \%$ of sevoflurane. In this experiment, triangulation is not the causative mechanism by which sevoflurane in higher concentrations induces TDP. 